\title{
Role of LOXL2 in the epithelial-mesenchymal transition and colorectal cancer metastasis
}

\author{
Pil-Gu Park ${ }^{1, *}$, Su Ji Jo ${ }^{1,2, *}$, Min Jung Kim ${ }^{1,3}$, Hyun Jeong Kim ${ }^{4}$ Ji Hae Lee ${ }^{5}$, Cheol \\ Keun Park6, Hyunki Kim6, Kang Young Lee?, Hoguen Kim ${ }^{2,6}$, Jeon Han Park ${ }^{1}$, Seung \\ Myung Dong ${ }^{5,8}$ and Jae Myun Lee ${ }^{1,2}$ \\ ${ }^{1}$ Department of Microbiology and Immunology, Yonsei University College of Medicine, Seoul, Republic of Korea \\ ${ }^{2}$ BK21 PLUS Project for Medical Science, Yonsei University College of Medicine, Seoul, Republic of Korea \\ ${ }^{3}$ Department of Pediatrics, Severance Hospital, Institute of Allergy, Yonsei University College of Medicine, Seoul, Republic \\ of Korea \\ ${ }^{4}$ Department of Nuclear Medicine, Yonsei University College of Medicine, Seoul, Republic of Korea \\ ${ }^{5}$ Research Institute, National Cancer Center, Goyang, Republic of Korea \\ ${ }^{6}$ Department of Pathology, Yonsei University, College of Medicine, Seoul, South Korea \\ ${ }^{7}$ Department of Surgery, Severance Hospital, Yonsei University College of Medicine, Seoul, Republic of Korea \\ ${ }^{8}$ IMK Bio-Convergence R\&D Center, International Vaccine Institute SNU Research Park, Seoul, Republic of Korea \\ *These authors contributed equally to this work
}

Correspondence to: Jae Myun Lee, email: jaemyun@yuhs.ac

Seung Myung Dong, email: smdongstar@gmail.com

Keywords: colorectal cancer, metastasis, LOXL2, epithelial-mesenchymal transition, tissue microarray analysis

Received: March 24, 2017

Accepted: May 11, 2017

Published: May 25, 2017

Copyright: Park et al. This is an open-access article distributed under the terms of the Creative Commons Attribution License 3.0 (CC BY

3.0), which permits unrestricted use, distribution, and reproduction in any medium, provided the original author and source are credited.

\section{ABSTRACT}

Colorectal cancer (CRC) is one of the most dangerous types of malignant tumors, and cancer metastasis is a major factor in the failure of CRC therapy. Recently, LOXL2 (lysyl oxidase-like 2) has been shown to represent a regulator of epithelialmesenchymal transition (EMT) in different cancer types. However, LOXL2 has not been reported to be involved in CRC metastasis. In this study, we demonstrated that LOXL2 expression is strongly correlated with the rate of CRC metastasis, it participates in the regulation of EMT-related molecule expression in CRC cells in vitro, and it is involved in migratory potential alterations. Additionally, tissue microarray analysis of CRC patients showed an increase in the probability of developing CRC distant metastasis and a decrease in the survival rate of patients with high LOXL2 expression. The results obtained in this study indicate that LOXL2 is involved in the development and progression of CRC metastasis, and therefore, its expression levels may represent a useful prognostic marker.

\section{INTRODUCTION}

Colorectal cancer $(\mathrm{CRC})$ is the third most common cancer worldwide $(\sim 10 \%$ of all cancer cases) and the second most prevalent cancer (2.4 million people) $[1,2]$. Approximately 700,000 people yearly die of CRC, and the development of metastatic disease is the most frequent cause of death $[1,3,4]$. The majority of CRC patients is diagnosed at the advanced cancer stage, and the risk of metastatic progression is increased in these patients [5]. Therefore, further studies should focus on the molecular mechanisms underlying the development of CRC metastasis, which may lead to the development of improved treatment strategies.

Epithelial-mesenchymal transition (EMT) is a molecular process essential for cancer metastasis initiation. During the process of EMT, cell properties are altered, and those required for migration, invasion 
into the extracellular matrix (ECM), and extravasation/ intravasation, are acquired $[6,7]$. In addition to these changes, the changes in the levels of many molecules can be observed, including several regulatory molecules that modulate the EMT.

Lysyl oxidase-like 2 (LOXL2) is a member of lysyl oxidase (LOX) family, which includes molecules sharing a highly conserved catalytic domain at the C-terminus [8]. In physiological conditions, LOXL2 is involved in the extracellular matrix (ECM) remodeling. Similar to the other members of LOX family, LOXL2 induces the crosslinking of ECM collagen and elastin by the deamination of peptidyl lysine residues $[8,9]$. Recently, several groups reported that LOXL2 may represent a modulator of EMT, through the regulation of EMT-inducing transcription factors, focal adhesion kinase (FAK)/Src signaling pathway, and the epigenetic regulation of EMT-related genes [10-12].

Furthermore, many studies, including ours, investigated and showed the clinical relevance of LOXL2 expression during the metastasis of breast [13], gastric [14], liver [15], esophageal [16], and pancreatic [17] cancers. However, LOXL2 in CRC has been rarely investigated [18, 19], and these studies focused on LOXL2 expression in association with $\mathrm{CRC}$ survival rate and tumor differentiation, and, to the best of our knowledge, the correlation between LOXL2 expression and the development of CRC metastasis has not been previously investigated. However, since the loss of the heterozygosity $(\mathrm{LOH})$ locus is frequently found in patients with colon cancer metastasis, and LOXL2 was reported to be one of the genes located at this locus [20], this may indicate a correlation between the expression levels of this molecule and the development of CRC metastasis. Therefore, we investigated the role of LOXL2 in the development and progression of CRC metastasis both in vitro and in patients.

\section{RESULTS}

\section{CRC cell migratory potential is associated with LOXL2 expression}

We first examined LOXL2 expression levels in several CRC cell lines. LOXL2 was shown to be expressed in SW480 cells, but rarely detected in other investigated cell lines, at mRNA and protein levels (Figure 1A and 1B). Although the expression of LOXL2 was determined to be lower than that determined in MDA-MB-231 cells, LOXL2-positive breast cancer cells [13], SW480 cells showed considerably higher migratory potential than LOXL2-negative cells, in migration assay and wound healing assay (Figure 1C and Supplementary Figure 1A). Cell migration rate was shown to be independent of the growth rate of different cells (Supplementary Figure 1B).

Next, we examined the migratory phenotype by using LOXL2 knockdown and ectopic expression in
A

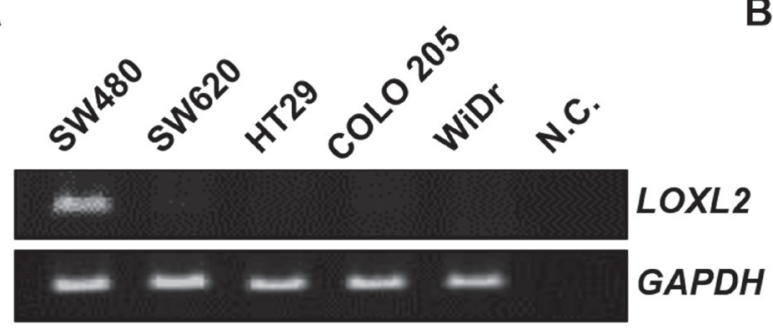

B

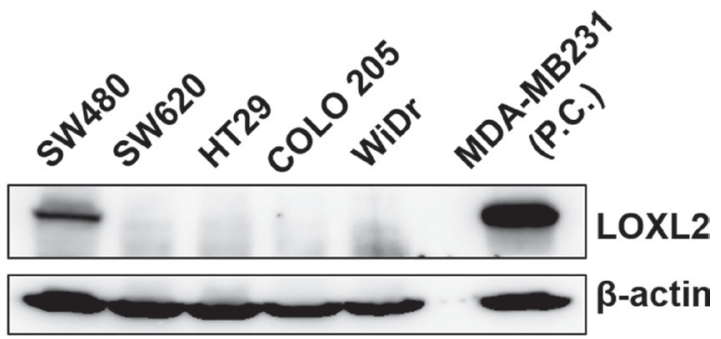

C

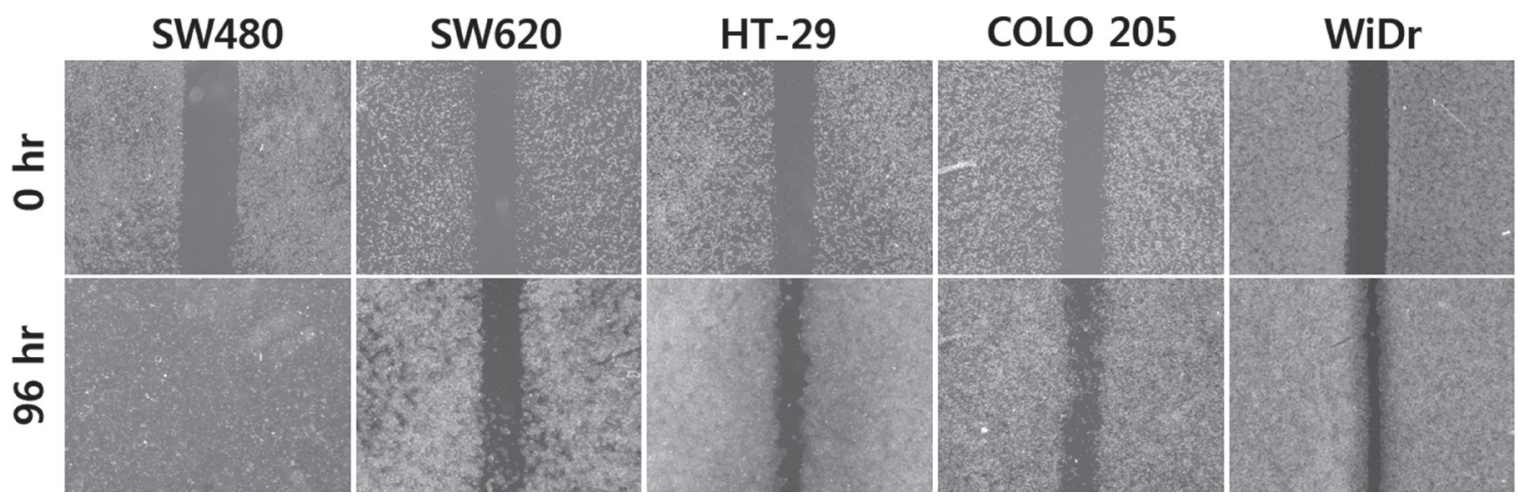

Figure 1: Correlation between LOXL2 expression levels and migratory potential of different CRC cells in vitro. (A) LOXL2 expression in five CRC cell lines. Distilled water was used as a negative control (N.C.). (B) LOXL2 protein levels in CRC cells. MDA-MB-231 was used as a positive control (P.C.). (C) Motility of different CRC cells in vitro, determined in wound healing assay. Representative figures obtained in three independent experiment are shown. 
SW480 and SW620 (LOXL2-negative) cells, respectively. We demonstrated that LOXL2 knockdown led to a significant reduction in the migratory potential of SW480 cells (Figure 2A and Supplementary Figure 2), while its ectopic expression led to the stimulation of SW620 cell migratory potential (Figure 2B).

Furthermore, we examined the ability of these cells to cross the endothelial cell barrier, represented by human umbilical vein endothelial cells (HUVEC), which mimics intra/extravasation process during metastasis. We observed that many SW480 cells were able to penetrate HUVEC cell layer, but $L O X L 2$ silencing led to a decrease in this potential (Figure 3A). SW620 cells transfected with the LOXL2 expression plasmid showed an increase in the HUVEC layer penetration, compared with that of the cells transfected with a mock plasmid (Figure 3B). Additionally, we confirmed that LOXL2 knockdown and overexpression did not affect cellular growth rates (Supplementary Figure 3).

\section{LOXL2 regulates the expression of EMT-related molecules in CRC cells}

In order to investigate the molecular mechanism underlying LOXL2-dependent alterations in migratory potential, we examined LOXL2 knockdown/ectopic expression-induced alterations in SW480 and SW620 cells, respectively (Figure 4A). We showed that LOXL2 knockdown leads to a decrease in the expression of vimentin, a mesenchymal marker, while LOXL2 overexpression upregulated the expression of this protein. Conversely, we obtained opposite effects on the expression of the epithelial marker, E-cadherin. Similar results were obtained at mRNA level (Figure 4B).

Furthermore, since the activation of FAK/Src pathway is known to promote EMT, several reports indicated that the changes in ECM stiffness can lead to the activation of FAK/Src signaling [21-23], and LOXL2 is known to be involved in the ECM remodeling, we investigated whether LOXL2 is involved in the activation of FAK/Src by determining the levels of phosphorylated FAK/Src (Figure 4C). LOXL2 knockdown and overexpression were shown to down- and upregulate, respectively, FAK/Src phosphorylation. Snail protein, one of the major transcription factors involved in the EMT process, was shown to be regulated by LOXL2, and LOXL2 knockdown led to a decrease, while its overexpression led to an increase in Snail levels (Figure 4C).

\section{Correlation between LOXL2 expression levels and clinicopathological characteristics of CRC patients}

We investigated the correlation between LOXL2 expression levels and CRC metastasis rate in patients, by immunohistochemically (IHC) examining LOXL2 expression in cancer tissue samples obtained from CRC patients. LOXL2 expression was classified as low or high (Figure 5A), and, of 223 patients examined in our

\section{A}
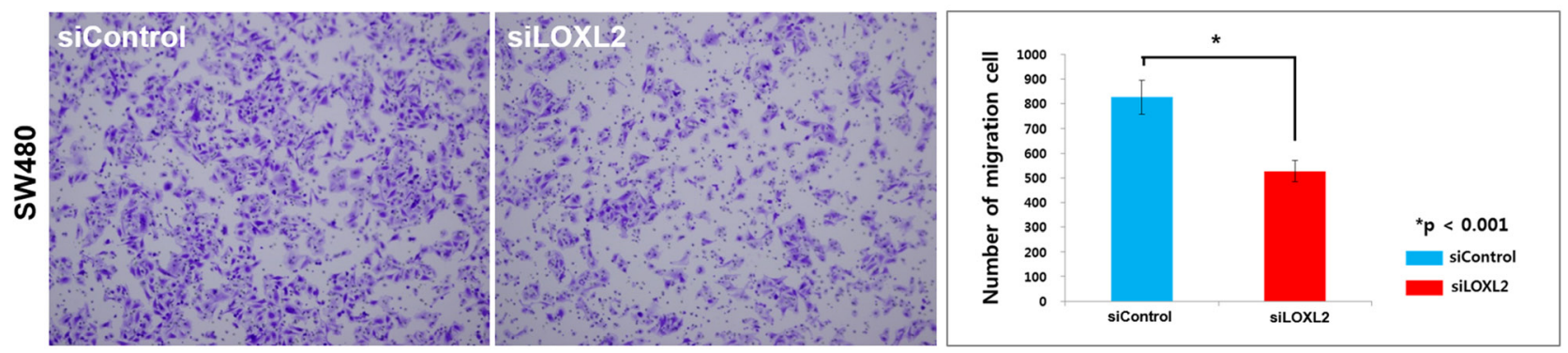

B
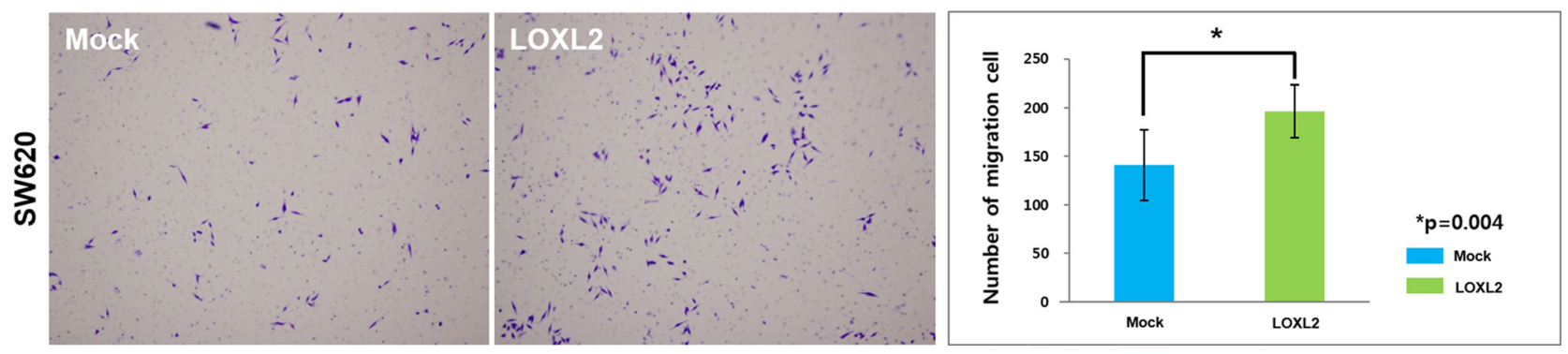

Figure 2: Migratory potential alterations induced by the changes in $L O X L 2$ expression. (A) Transwell migration of $L O X L 2-$ knockdown SW480 cells. Images were obtained $36 \mathrm{~h}$ after seeding. (B) Transwell migration of LOXL2-overexpressing SW620 cells. Images were obtained $72 \mathrm{~h}$ after seeding. Results were obtained from three independent experiments, and bar graphs in (A) and (B) represent cell number per image field (mean \pm standard deviation). 
study, the samples obtained from 28 patients (12.1\%) were shown to have high LOXL2 expression levels, which was shown to be significantly associated with the presence of distant metastasis $(p=0.046)$. Increased LOXL2 expression levels tended to be associated with the survival of the patients $(p=0.066)$. Other clinicopathological parameters, including patient age, sex, histological grade, pathologic T stage, overall cancer stage, and the presence of lymph node metastasis were not associated with LOXL2 expression ( $p>0.05$ for all). Clinicopathological characteristics in patients categorized by LOXL2 expression levels are summarized in Table 1. Patients with high LOXL2 expression levels were shown to have a shorter overall survival ( $p=0.024$; Figure $5 \mathrm{~B}$ ) than those with low LOXL2 expression.

\section{DISCUSSION}

Here, we investigated the role of LOXL2 in the EMT progression in $\mathrm{CRC}$, and clinical consequences of the changes in LOXL2 expression. We observed that LOXL2-positive CRC cells have an increased migratory potential, in comparison with that of the LOXL2-negative cells; LOXL2 knockdown/ectopic expression was shown to affect this migratory potential in these cells in vitro, demonstrating that LOXL2 promotes the EMT process, while the results obtained by analyzing clinicopathological parameters strongly support the pro-metastatic role of LOXL2 in CRC progression.

Various molecular mechanisms underlying LOXL2dependent EMT induction have been reported. Peinado et al. [10] demonstrated that LOXL2 stabilizes Snail, one of the major transcription factors inducing EMT process, by oxidizing lysine 98 and 137 residues, and this process and the phosphorylation of Snail by glycogen synthase kinase 3 beta (GSK3 $\beta$ ), which is a signal for proteasomedependent degradation, are considered antagonistic processes. Here, we demonstrated that the changes in LOXL2 expression affect Snail at protein, but not at mRNA level, suggesting that LOXL2 modulates SNAI1 at the post-translation levels, which further affects the migratory potential of CRC cells in vitro.

Other members of LOX family have been suggested to induce the activation of EMT, including LOX, which was reported to be involved in the development of CRC metastasis [24], through the activation of FAK/Src signaling pathway, which induces the EMT process, and the activation of the pathway induced by ECM remodeling, which may trigger the activation of cellular receptors, such as integrin. Previously, several studies reported that LOXL2 induces EMT in breast, gastric, and pancreatic cancer cells, which is accompanied by the activation of

A
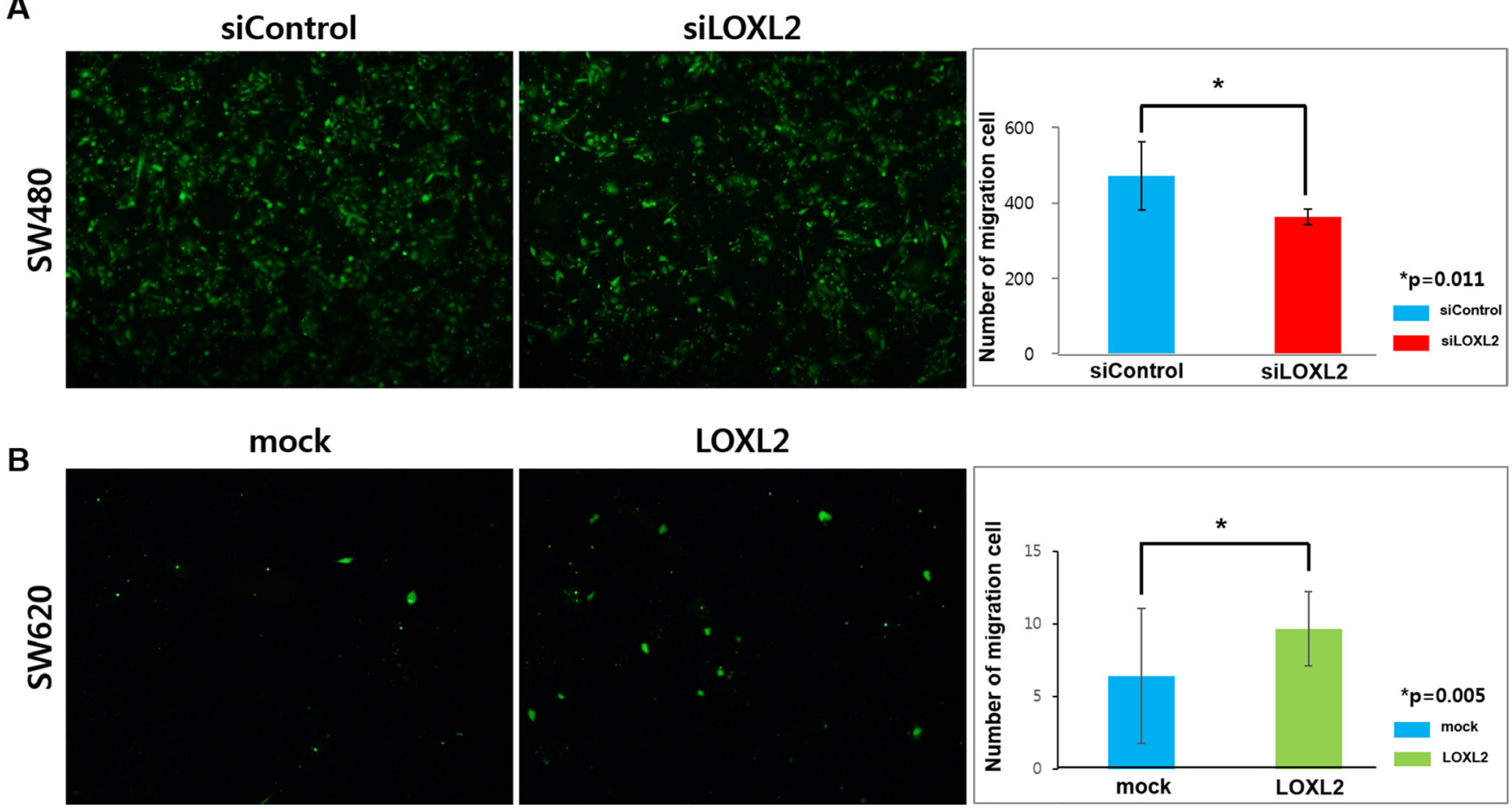

Figure 3: Effects of $L O X L 2$ silencing/ectopic expression on the migration of CRC cells through the endothelial barrier. (A) SW480 cells transfected with siControl/siLOXL2 oligonucleotide were seeded on HUVECs, and images were obtained $36 \mathrm{~h}$ after seeding. (B) SW620 cells transfected with mock/LOXL2-expressing plasmids were seeded on HUVECs, and images were obtained $72 \mathrm{~h}$ after seeding. Results were obtained from three independent experiments and bar graphs in (A) and (B) represent cell number per image field (mean \pm standard deviation). 
Table 1: Clinicopathological characteristics of CRC patients categorized based on their LOXL2 expression levels

\begin{tabular}{|c|c|c|c|c|c|c|c|}
\hline \multirow{2}{*}{$\begin{array}{l}\text { Category } \\
\text { Age }\end{array}$} & \multirow{2}{*}{$\begin{array}{l}\text { Variables } \\
<60\end{array}$} & \multirow{2}{*}{$\begin{array}{c}\begin{array}{c}\text { No. of cases } \\
(\boldsymbol{n}=\mathbf{2 2 3})\end{array} \\
97\end{array}$} & \multicolumn{2}{|c|}{$\begin{array}{l}\text { High expression }(\%) \\
\qquad(n=28)\end{array}$} & \multicolumn{2}{|c|}{$\begin{array}{l}\text { Low expression }(\%) \\
\qquad(n=195)\end{array}$} & \multirow{2}{*}{$\frac{p \text {-value }}{0.942}$} \\
\hline & & & 12 & $(42.9)$ & 85 & $(43.6)$ & \\
\hline & $\geq 60$ & 126 & 16 & $(57.1)$ & 110 & $(56.4)$ & \\
\hline \multirow[t]{2}{*}{ Gender } & Female & 81 & 13 & $(46.4)$ & 68 & $(34.9)$ & 0.235 \\
\hline & Male & 142 & 15 & $(53.6)$ & 127 & $(65.1)$ & \\
\hline \multirow[t]{2}{*}{ Location } & Colon & 112 & 13 & $(46.4)$ & 99 & $(50.8)$ & 0.668 \\
\hline & Rectum & 111 & 15 & $(53.6)$ & 96 & $(49.2)$ & \\
\hline \multirow[t]{2}{*}{ Histologic grade } & WD to MD & 205 & 25 & $(89.3)$ & 180 & $(92.3)$ & 0.584 \\
\hline & PD & 18 & 3 & $(10.7)$ & 15 & $(7.7)$ & \\
\hline \multirow[t]{2}{*}{ Pathologic T stage } & $\mathrm{T} 1$ and $\mathrm{T} 2$ & 30 & 3 & $(10.7)$ & 27 & $(13.8)$ & 0.650 \\
\hline & $\mathrm{T} 3$ and $\mathrm{T} 4$ & 193 & 25 & $(89.3)$ & 168 & $(86.2)$ & \\
\hline \multirow[t]{2}{*}{ LNM } & Absent & 103 & 11 & $(39.3)$ & 92 & $(47.2)$ & 0.434 \\
\hline & Present & 120 & 17 & $(60.7)$ & 103 & $(52.8)$ & \\
\hline \multirow[t]{2}{*}{ Distant metastasis } & Absent & 188 & 20 & $(71.4)$ & 168 & $(86.2)$ & 0.046 \\
\hline & Present & 35 & 8 & $(28.6)$ & 27 & $(13.8)$ & \\
\hline \multirow[t]{4}{*}{ Overall cancer stage } & I & 23 & 1 & (3.6) & 22 & (11.3) & 0.104 \\
\hline & II & 73 & 9 & $(32.1)$ & 64 & $(32.8)$ & \\
\hline & III & 92 & 10 & $(35.7)$ & 82 & $(42.1)$ & \\
\hline & IV & 35 & 8 & $(28.6)$ & 27 & (13.8) & \\
\hline \multirow[t]{2}{*}{ Survival } & Alive & 146 & 14 & $(50.0)$ & 132 & $(67.7)$ & 0.066 \\
\hline & Expired & 77 & 14 & $(50.0)$ & 63 & (32.3) & \\
\hline
\end{tabular}

WD: well differentiated; MD: moderately differentiated; PD: poorly differentiated; LNM: lymph node metastasis.

FAK/Src pathway $[13,14,17]$. This may suggest that all members of LOX family induce EMT through ECM remodeling. However, LOXL2 may play the key role in this process, because this is the only member of LOX family that can stabilize Snail [10]. Here, we observed that LOXL2 induces FAK/Src activation and regulates Snail in vitro, and therefore, we suggest that LOXL2 may have dual effects on the EMT activation in CRC cells.
A

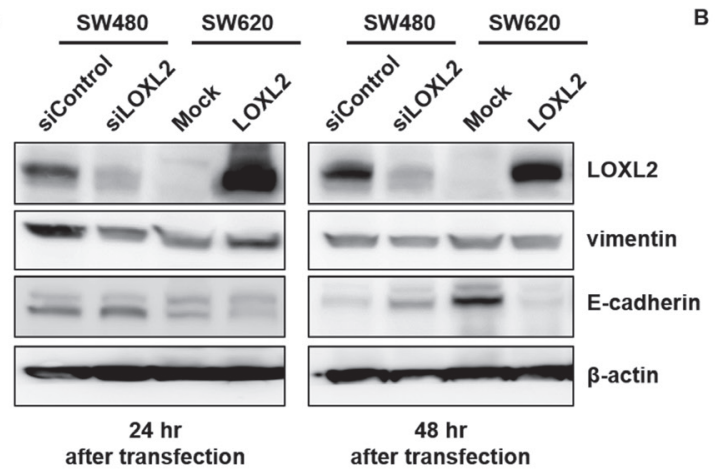

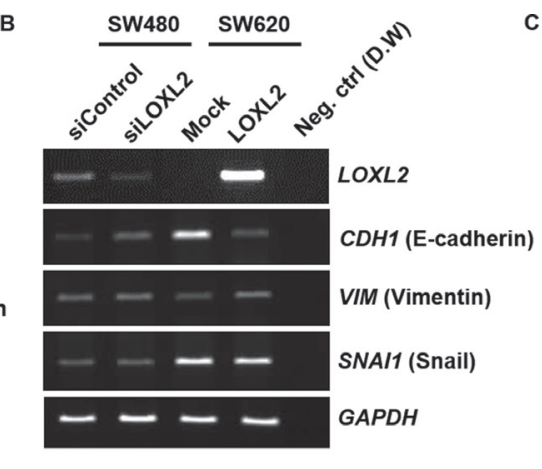

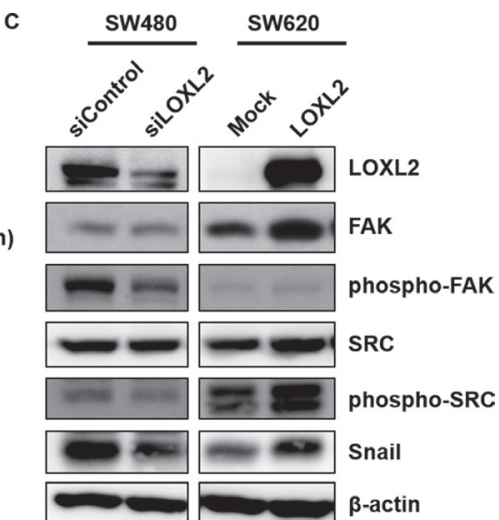

Figure 4: Induction of EMT by LOXL2 through the activation of FAK/Src pathway and upregulation of Snail. (A) Expression of EMT-related molecules 24 and $48 \mathrm{~h}$ after the transfection of cells with LOXL2 siRNA/overexpressing plasmid. (B) CDH1 (E-cadherin), VIM (vimentin), and SNAI1 (Snail) expression in SW480 and SW620 cells 24 h after the transfection with siLOXL2 and LOXL2-overexpressing plasmid, respectively. GAPDH was used as an internal control. (C) Expression of EMT-related molecules in cells transfected with siLOXL2 and LOXL2-overexpressing plasmid. 
Metastatic cells generally have a higher migratory potential compared with that of the primary tumor cells. SW480 and SW620 cell lines have originated from one patient. The former was isolated from a primary colorectal cancer and the latter from a lymph node metastasis [25]. However, the results obtained here demonstrated that SW480 cells show higher motility and migratory potential than SW620 in vitro. Previously, different studies reported controversial results when analyzing migratory potential of these cells [25-35], which may be due to the alterations in cell line characteristic during long-term in vitro culturing, or it is possible that SW620 cells underwent mesenchymal-epithelial transition (MET), in order to establish and grow at the distant metastatic sites [36, 37].

In spite of inverted migratory capacity to conceptual expectation, it is possible that SW620 possesses higher metastatic potential than SW480 in vivo. It was reported in several studies that SW620 cells show an increased metastatic potential in xenograft experiments compared with that of SW480 cells $[38,39]$. Due to the complexity of metastatic process, other factors, such as proteolytic potential and cancer cell stemness are crucial for this process as well $[6,40]$, and some of these factors may be responsible for the observed discrepancy between the migratory capacity and metastatic potential of two analyzed cell lines. Although high migratory potential is not sufficient for the development of metastasis, it is necessary, and therefore, our results strongly indicate that LOXL2 plays crucial role in the development of CRC metastasis.

Although previous reports suggested that LOXL2 expression may be related to CRC differentiation and stage $[18,19]$, the results of our clinical analysis showed a correlation between CRC metastasis rate and LOXL2 expression, which agrees with the results obtained in studies investigating LOXL2 roles in other cancer types [13-17], and the study examining the LOH locus in CRC patients with liver metastasis [20]. Among 13 genes at the LOH locus, LOXL2 expression was shown to be increased in patients with liver metastasis, compared with that in the normal tissues or matched primary cancer by locus mutation, which supports our conclusion that LOXL2 induces EMT/metastasis. Furthermore, LOXL2 expression was shown to be associated with patient survival rate, suggesting that the levels of this molecule may serve as a prognostic marker in CRC.

Currently, different characteristics are used in clinic for the classification of CRCs. Microsatellite

A

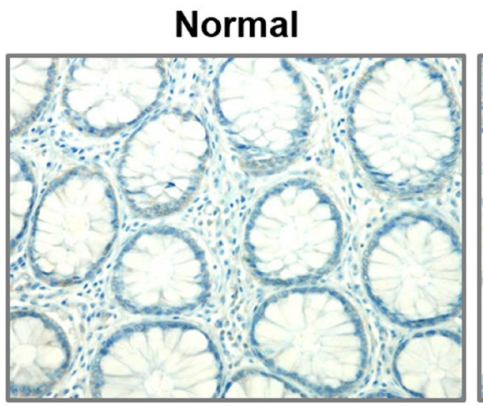

Low expression

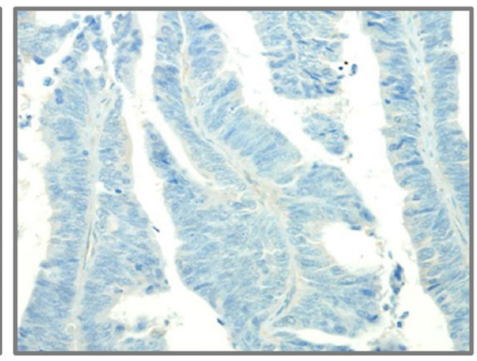

B

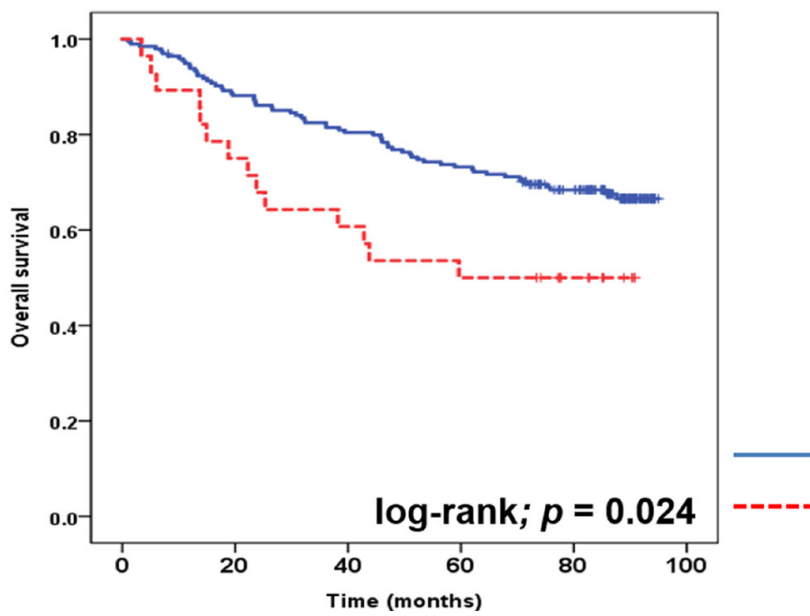

\section{Low LOXL2 expression ( $n=195)$}

High LOXL2 expression $(n=28)$

Figure 5: LOXL2 IHC analysis in CRC tissues and the correlation between this parameter and the survival of CRC patients. (A) IHC analysis of LOXL2 expression in CRC tissues. Representative images of normal colon tissue, CRC tissue with low LOXL2 expression, and CRC tissue with high LOXL2 expression are presented (magnification, $200 \times$ ). (B) Correlation between the overall survival rates of CRC patients and LOXL2 expression. High LOXL2 expression was correlated with poor patient survival (log-rank MantelCox test, $p=0.024)$. 
instability (MSI), one of the essential factors used for the classification of CRCs, occurs due to the deficiencies in mismatch repair (MMR) mechanism, believed to represent a main cause of carcinogenesis [41, 42]. Clinically, five to eight specific loci in CRC patients are investigated in order to establish the diagnosis of MSI- or microsatellite stable (MSS)-CRC, and this process is crucial for the selection of therapeutic strategies [43]. While it is believed that MSICRCs have a more positive prognosis and lower frequency of metastasis [44], 80 85\% of CRCs are diagnosed as MSS-CRCs [45], requiring further molecular studies of the mechanism of carcinogenesis and metastasis process. Here, we used MSS-CRC cells, since only six patients (2.7\%) were diagnosed as MSI-CRC in TMA analysis performed in this study (data not shown), but all screened cell lines were of MSS-CRC origin.

In conclusion, the results obtained in our study demonstrated that LOXL2 expression levels may be of clinical significance in $\mathrm{CRC}$, and the mechanisms underlying the effects of this molecule include the activation of FAK/Src signaling and the stabilization of Snail. The analysis of clinicopathological parameters of CRC patients demonstrated that LOXL2 may represent a valuable prognostic marker for these patients. Furthermore, molecular mechanisms underlying LOXL2-mediated EMT induction in $\mathrm{CRC}$, which we elucidated here, may lead to the development of improved therapeutic strategies for the treatment of CRC metastasis. Targeting of transcription factors or signaling molecules associated with the process of EMT may generally have harmful effects, due to the presence and functions of these molecules in the healthy tissues. However, LOXL2 is generally not expressed in normal colon tissues [46], which may allow a specific targeting of CRC metastatic cells, and, since LOXL2 is an enzyme, its potential inhibitors can be easily and rapidly screened. The clinical application of several inhibitors targeting LOXL2 is being investigated currently, although not for the treatment of CRC $[47,48]$. Taken together, our results and previous reports demonstrate that LOXL2 may represent a promising therapeutic target for the treatment of metastatic CRC.

\section{MATERIALS AND METHODS}

\section{Cell culture}

All CRC cell lines (SW480, SW620, HT-29, COLO 205, and WiDr) were purchased from the American Type Culture Collection (ATCC, Manassas, VA, USA), and cultured in Dulbecco's Modified Eagle Medium (DMEM; Hyclone, Logan, UT, USA) supplemented with 10\% fetal bovine serum (FBS; Hyclone) and $10 \mathrm{U} / \mathrm{mL}$ of penicillinstreptomycin (Hyclone). All cell lines were routinely tested for mycoplasma contamination and cell lines used in subsequent experiments (SW480 and SW620) were authenticated by short tandem repeat profiling at the
Research Institute of National Cancer Center (Republic of Korea).

HUVEC cells were purchased from ATCC, and cultured in Endothelial Cell Growth medium (EGM-2; Lonza, Walkersville, MD, USA) supplemented with $10 \%$ FBS.

\section{Knockdown and ectopic expression of LOXL2}

For LOXL2 knockdown, cells were transfected with small interfering (si)RNA targeting LOXL2 (siLOXL2; 5'-GCCACAUAGGUGGUUCCUUCAUU-3') or non specific oligonucleotide (siControl; 5'-CGUUAAUCG CGUAUAAUACGCGUA-3') using Lipofectamine RNAiMAX (Invitrogen/Life Technologies, Green Island, NY, USA), according to the manufacturer's instructions.

For the ectopic expression of LOXL2, cells were transfected with LOXL2-expressing plasmid (pcDNA3.1(+)-hLOXL2 [17]) using Lipofectamine 2000 (Invitrogen/Life Technologies) according to the manufacturer's instructions.

\section{Reverse transcription-polymerase chain reaction (RT-PCR)}

Total RNA was extracted from CRC cells using TRIZOL reagent (Gibco BRL/Life Technologies, Green Island, NY, USA), and cDNA was subsequently synthesized using SuperScriptIII First-Strand Synthesis System (Invitrogen/Life Technologies), according to the manufacturer's instructions. Synthesized cDNAs were used as a template for subsequent PCR, determining the levels of SNAI1, LOXL2, VIM, CDH1, and GAPDH. Primer sequences used in each PCR set are shown in Supplementary Table 1.

\section{Western blot analysis}

Cells were lysed with radioimmunoprecipitation assay (RIPA) buffer (Sigma-Aldrich, St. Louis, MO, USA) supplemented with protease inhibitors and sampled in protein sample buffer $(250 \mathrm{mM}$ Tris-Cl, 1.6 mM EDTA, 8\% sodium dodecyl sulfate (SDS), 40\% glycerol, $0.02 \%$ Pyronin Y dye, 1.5\% 2'-mercaptoethanol). Protein samples were separated using 8-10\% SDSpolyacrylamide gel electrophoresis and transferred onto nitrocellulose membranes, which were blocked and probed with antibodies against LOXL2 (Origene, Rockville, MD, USA), SNAI1 (Cell Signaling, Danvers, MA, USA), phosphor-FAK; Tyr576, and FAK (Santa Cruz Biotechnology, CA, USA), phosphor-Src;Tyr418 (Millipore, Billerica, MA, USA), Src (Santa Cruz Biotechnology), CDH1 (BD Biosciences, Sparks, MD), VIM (Dako, Glostrup, Denmark), and $\beta$-actin (SigmaAldrich). The blots were prepared using WesternBright Sirius solution (Advansta; Menlo Park, CA, USA) and 
analyzed with enhanced chemiluminescence using ImageQuant LAS Chemi-luminescent Image Analyzer (GE Healthcare/Life Technologies, Green Island, NY, USA).

\section{Wound-healing assay}

In order to induce a wound in a monolayer of CRC cells, a culture insert (IBIDI, Martinsried, Germany), consisting of two reservoirs separated by a $500-\mu \mathrm{m}$ thick wall, was placed into each well of six-well plates, and $5 \times 10^{4}$ cells were added into the two reservoirs. After 24 $\mathrm{h}$ of incubation at $37^{\circ} \mathrm{C}$, the insert was gently removed, and the cells were allowed to migrate for 5 days. Images were taken every $24 \mathrm{~h}$ using Olympus IX71 inverted microscope equipped with a digital camera (Olympus France, Rungis, France).

For the comparative analysis of migratory potential according to LOXL2 expression in SW480 cells, control SW480 cells and LOXL2-silenced SW480 cells $\left(5 \times 10^{4}\right.$ cells/well) were seeded into 96-well Essen ImageLock microplates (Essen BioScience, Ann Arbor, MI, USA), and incubated at $37^{\circ} \mathrm{C}$ for $18 \mathrm{~h}$. Cell monolayers were scratched using a 96-well Wound Maker (Essen BioScience), and the wells were washed with phosphate-buffered saline (PBS) to remove the detached cells. Images of the wounds were automatically recorded at every $6 \mathrm{~h}$ for $96 \mathrm{~h}$ using IncuCyte ZOOM software (Essen BioScience).

\section{Transwell migration and trans-endothelial migration assays}

The migratory capacity of CRC cells was assessed in vitro using transwell migration chambers (Corning, NY, USA) in accordance with the manufacturer's instructions. Briefly, cells were serum-starved for $24 \mathrm{~h}$, then detached by brief trypsinization, and resuspended in the serum-free medium. For each well, $2-4 \times 10^{5}$ cells were seeded onto $0.1 \%$ gelatin-coated insert in the transwell chamber $(8-\mu \mathrm{m}$ pore size and $6.5-\mathrm{mm}$ diameter), and the inserts were set in chambers filled with $400 \mu \mathrm{L}$ of complete medium. Cells were incubated in a $\mathrm{CO}_{2}$ incubator at $37^{\circ} \mathrm{C}$ for 36 or $72 \mathrm{~h}$, and the inserts were fixed with methanol and stained with hematoxylin-eosin solution (Sigma-Aldrich). Following the staining, the upper surface of membranes, containing the remaining non-migrated cells, was wiped with cotton swabs, and the lower membrane surfaces, containing cells that migrated, were mounted onto slides. Images were taken using Olympus IX71 inverted microscope equipped with a digital camera (Olympus, France).

For trans-endothelial migration assay, $3 \times 10^{4}$ HUVECs were seeded onto $0.1 \%$ gelatin-coated insert $24 \mathrm{~h}$ prior to the seeding of 2-4 $\times 10^{5}$ carboxyfluorescein succinimidyl ester-labeled (CFSE; Thermo Fisher, Waltham, MA, USA) cells resuspended in serum-free medium.
The inserts were set in the wells filled with the complete medium. After 36 or $72 \mathrm{~h}$ of incubation, the upper surface of the membranes was wiped with cotton swabs and the migrated cells on the lower surface fixed and mounted onto slides. Images were taken using Olympus DP71 fluorescent microscope equipped with a digital camera (Olympus France, Rungis, France) with green fluorescent protein (GFP) filter, in order to visualize CFSE-labeled cells.

All experiments were performed three times and, in each experiment, images were taken in 4-5 random fields per slide at $100 \times$ magnification.

\section{Cell proliferation assay}

For the comparison of proliferation rates between five investigated CRC cell lines, $5 \times 10^{4}$ cells of each line were seeded into 12 -well plates and cell numbers were manually determined using disposable hemocytometers every $24 \mathrm{~h}$.

For the proliferation assay with $L O X L 2$-knockdown/ overexpression cells, Cell Counting Kit-8 (Dojindo Molecular Technologies Inc, Rockville, MD, USA) was used, according to the manufacturer's instructions. Relative proliferation rates were calculated by detecting absorbance at $450 \mathrm{~nm}$ using Epoch Microplate Spectrophotometer (BioTek Instruments Inc, Winooski, VT, USA).

\section{CRC patient selection and tissue microarray construction}

Patients who underwent surgical resection due to CRC in 2003 and 2004 at Severance Hospital (Seoul, Republic of Korea) were included in this study. Patients treated with neoadjuvant chemoradiation therapy, which may affect the EMT process [49], were excluded. Various clinicopathologic factors, such as the age at operation, gender, size and location of tumor, and clinical follow-up data were obtained from the review of medical records. Overall survival time was estimated from the date of curative resection to the date of the last follow-up or death from any cause.

Pathologic factors, such as histologic grade of tumor and pathologic TNM staging according to the $7^{\text {th }}$ American Joint Committee on Cancer criteria were obtained from the slides independently reviewed by two pathologists (C. K. Park and H. Kim). Two cores of representative tumor area and one core of the representative normal area were extracted from each case for tissue microarray construction as previously described [13]. This study was approved by the Institutional Review Board of Severance Hospital, Seoul, Republic of Korea (IRB 4-2012-0026), and the study was conducted according to the principles expressed in the Declaration of Helsinki. 


\section{IHC staining and analysis}

Four-micrometer large tissue sections from the tissue microarray recipient blocks were used for IHC, which was performed using the Ventana Discovery XT automated staining system (Ventana Medical Systems, Inc., Tucson, AZ, USA) and anti-LOXL2 antibody (Origene).

Immunostained slides were evaluated by two pathologists (C. K. Park and H. Kim) independently. LOXL2 expression was categorized as negative, focal positive, and positive. Further, these samples were considered as low expression samples if they were negative or focal positive, while positive samples were considered high expression samples.

\section{Statistical analysis}

For transmigration assay and trans-endothelial migration assay, statistical analyses and the determination of $p$-values were performed by using Student's $t$-test, with IBM SPSS Statistics software v. 23 (SPSS Inc., Chicago, IL, USA).

For the analysis of clinicopathological characteristics, SPSS for Windows version 21.0 (SPSS Inc.) was used. Mann-Whitney $U$-test and Fisher's exact test were used for the analysis of various clinicopathologic factors. $p$-values $<0.05$ were considered statistically significant. Kaplan-Meier survival curves with log-rank statistics were applied to evaluate time to survival.

\section{Abbreviations}

CRC, colorectal cancer; EMT, epithelialmesenchymal transition; ECM, extracellular matrix; FAK, focal adhesion kinase; GSK3 $\beta$, glycogen synthase kinase 3 beta; HUVEC, human umbilical vein endothelial cell; IHC, immunohistochemistry; LOH, loss of the heterozygosity; LOX, lysyl oxidase; LOXL2, lysyl oxidase-like 2.

\section{Authors' contributions}

Prof. Jae Myun Lee and Dr. Seung Myung Dong were responsible for the conception and design of the study, and data interpretation. Pil-gu Park, Su Ji Jo, and Ji Hae Lee performed molecular experiments and were involved in data acquisition and analysis. Prof. Hoguen Kim, Prof. Hyunki Kim, and Cheol Keun Park performed clinicopathologic analyses. Cheol Keun Park, Min Jung Kim, and Prof. Hyun Jeong Kim performed statistical analysis. Prof. Jeon Han Park performed a critical revision of the experiments and manuscript. Prof. Kang Young Lee provided TMA slides of CRC patients.

\section{ACKNOWLEDGMENTS}

The authors want to thank Keun Bae Bang (Department of Pathology, Yonsei University, College of
Medicine, Seoul, Korea) for the technical support with immunohistochemistry and Mi Kyung Song (Biostatistics Collaboration Unit, Yonsei University College of Medicine) for the advice with the statistical analysis.

\section{CONFLICTS OF INTEREST}

The authors have declared no conflicts of interest in relation to this manuscript.

\section{FUNDING}

This work was supported by the National Research Foundation of Korea (NRF) grant funded by the Korea government (NRF-2015R1A2A2A01005412) and by a faculty research grant of Yonsei University College of Medicine (6-2009-0150).

\section{REFERENCES}

1. Torre LA, Bray F, Siegel RL, Ferlay J, Lortet-Tieulent J, Jemal A. Global cancer statistics, 2012. CA Cancer J Clin. 2015; 65:87-108.

2. Parkin DM. Global cancer statistics in the year 2000. Lancet Oncol. 2001; 2:533-543.

3. Simmonds PC, and Colorectal Cancer Collaborative Group. Palliative chemotherapy for advanced colorectal cancer: systematic review and meta-analysis. BMJ. 2000; 321:531-35.

4. Shimada H, Tanaka K, Endou I, Ichikawa Y. Treatment for colorectal liver metastases: a review. Langenbecks Arch Surg. 2009; 394:973-983.

5. Clarke N, McDevitt J, Kearney PM, Sharp L. Increasing late stage colorectal cancer and rectal cancer mortality demonstrates the need for screening: a population based study in Ireland, 1994-2010. BMC Gastroenterol. 2014; 14:92.

6. Wan L, Pantel K, Kang Y. Tumor metastasis: moving new biological insights into the clinic. Nat Med. 2013; 19:1450-1464.

7. Kalluri R, Weinberg RA. The basics of epithelialmesenchymal transition. J Clin Invest. 2009; 119:14201428.

8. Barker HE, Cox TR, Erler JT. The rationale for targeting the LOX family in cancer. Nat Rev Cancer. 2012; 12:540-552.

9. Moon HJ, Finney J, Ronnebaum T, Mure M. Human lysyl oxidase-like 2. Bioorg Chem. 2014; 57:231-241.

10. Peinado H, Del Carmen Iglesias-de la Cruz M, Olmeda D, Csiszar K, Fong KS, Vega S, Nieto MA, Cano A, Portillo F. A molecular role for lysyl oxidase-like 2 enzyme in snail regulation and tumor progression. EMBO J. 2005; 24:3446-3458.

11. Millanes-Romero A, Herranz N, Perrera V, Iturbide A, Loubat-Casanovas J, Gil J, Jenuwein T, Garcia de Herreros A, Peiro S. Regulation of heterochromatin transcription by 
Snail1/LOXL2 during epithelial-to-mesenchymal transition. Mol Cell. 2013; 52:746-757.

12. Cuevas EP, Moreno-Bueno G, Canesin G, Santos V, Portillo F, Cano A. LOXL2 catalytically inactive mutants mediate epithelial-to-mesenchymal transition. Biol Open. 2014; 3:129-137.

13. Ahn SG, Dong SM, Oshima A, Kim WH, Lee HM, Lee SA, Kwon SH, Lee JH, Lee JM, Jeong J, Lee HD, Green JE. LOXL2 expression is associated with invasiveness and negatively influences survival in breast cancer patients. Breast Cancer Res Treat. 2013; 141:89-99.

14. Peng L, Ran YL, Hu H, Yu L, Liu Q, Zhou Z, Sun YM, Sun LC, Pan J, Sun LX, Zhao P, Yang ZH. Secreted LOXL2 is a novel therapeutic target that promotes gastric cancer metastasis via the Src/FAK pathway. Carcinogenesis. 2009; 30:1660-1669.

15. Wong CC, Tse AP, Huang YP, Zhu YT, Chiu DK, Lai RK, Au SL, Kai AK, Lee JM, Wei LL, Tsang FH, Lo RC, Shi J, et al. Lysyl oxidase-like 2 is critical to tumor microenvironment and metastatic niche formation in hepatocellular carcinoma. Hepatology. 2014; 60:1645-1658.

16. Li TY, Xu LY, Wu ZY, Liao LD, Shen JH, Xu XE, Du ZP, Zhao Q, Li EM. Reduced nuclear and ectopic cytoplasmic expression of lysyl oxidase-like 2 is associated with lymph node metastasis and poor prognosis in esophageal squamous cell carcinoma. Hum Pathol. 2012; 43:1068-1076.

17. Park JS, Lee JH, Lee YS, Kim JK, Dong SM, Yoon DS. Emerging role of LOXL2 in the promotion of pancreas cancer metastasis. Oncotarget. 2016; 7:42539-42552. https://doi.org/10.18632/oncotarget.9918.

18. Torres S, Garcia-Palmero I, Herrera M, Bartolome RA, Pena C, Fernandez-Acenero MJ, Padilla G, PelaezGarcia A, Lopez-Lucendo M, Rodriguez-Merlo R, Garcia de Herreros A, Bonilla F, Casal JI. LOXL2 Is Highly Expressed in Cancer-Associated Fibroblasts and Associates to Poor Colon Cancer Survival. Clin Cancer Res. 2015; 21:4892-4902.

19. Fong SF, Dietzsch E, Fong KS, Hollosi P, Asuncion L, He Q, Parker MI, Csiszar K. Lysyl oxidase-like 2 expression is increased in colon and esophageal tumors and associated with less differentiated colon tumors. Genes Chromosomes Cancer. 2007; 46:644-655.

20. Macartney-Coxson DP, Hood KA, Shi HJ, Ward T, Wiles A, O'Connor R, Hall DA, Lea RA, Royds JA, Stubbs RS, Rooker S. Metastatic susceptibility locus, an 8p hot-spot for tumour progression disrupted in colorectal liver metastases: 13 candidate genes examined at the DNA, mRNA and protein level. BMC Cancer. 2008; 8:187.

21. Canel M, Serrels A, Frame MC, Brunton VG. E-cadherinintegrin crosstalk in cancer invasion and metastasis. J Cell Sci. 2013; 126(Pt 2):393-401.

22. Lu P, Weaver VM, Werb Z. The extracellular matrix: a dynamic niche in cancer progression. J Cell Biol. 2012; 196:395-406.
23. Lopez JI, Mouw JK, Weaver VM. Biomechanical regulation of cell orientation and fate. Oncogene. 2008; 27:6981-6993.

24. Baker AM, Cox TR, Bird D, Lang G, Murray GI, Sun XF, Southall SM, Wilson JR, Erler JT. The role of lysyl oxidase in SRC-dependent proliferation and metastasis of colorectal cancer. J Natl Cancer Inst. 2011; 103:407-424.

25. Leibovitz A, Stinson JC, McCombs WB 3rd, McCoy CE, Mazur KC, Mabry ND. Classification of human colorectal adenocarcinoma cell lines. Cancer Res. 1976; 36:4562-4569.

26. Luo F, Li J, Wu S, Wu X, Chen M, Zhong X, Liu K. Comparative profiling between primary colorectal carcinomas and metastases identifies heterogeneity on drug resistance. Oncotarget. 2016; 7:63937-63949. https://doi. org/10.18632/oncotarget.11570.

27. Fang Y, Sun B, Xiang J, Chen Z. MiR-301a promotes colorectal cancer cell growth and invasion by directly targeting SOCS6. Cell Physiol Biochem. 2015; 35:227-236.

28. Liu Y, Zhou Y, Feng X, An P, Quan X, Wang H, Ye S, Yu C, He Y, Luo H. MicroRNA-126 functions as a tumor suppressor in colorectal cancer cells by targeting CXCR4 via the AKT and ERK1/2 signaling pathways. Int J Oncol. 2014; 44:203-210.

29. Sun HB, Chen X, Ji H, Wu T, Lu HW, Zhang Y, Li H, Li YM. miR494 is an independent prognostic factor and promotes cell migration and invasion in colorectal cancer by directly targeting PTEN. Int J Oncol. 2014; 45:2486-2494.

30. Chen ML, Liang LS, Wang XK. miR-200c inhibits invasion and migration in human colon cancer cells SW480/620 by targeting ZEB1. Clin Exp Metastasis. 2012; 29:457-469.

31. Feng J, Fu Z, Guo J, Lu W, Wen K, Chen W, Wang H, Wei J, Zhang S. Overexpression of peroxiredoxin 2 inhibits TGF-beta1induced epithelial-mesenchymal transition and cell migration in colorectal cancer. Mol Med Rep. 2014; 10:867-873.

32. Yuan Z, Yu X, Ni B, Chen D, Yang Z, Huang J, Wang J, Wang L. Overexpression of long non-coding RNACTD903 inhibits colorectal cancer invasion and migration by repressing Wnt/beta-catenin signaling and predicts favorable prognosis. Int J Oncol. 2016; 48:2675-2685.

33. Ratke J, Entschladen F, Niggemann B, Zanker KS, Lang K. Leptin stimulates the migration of colon carcinoma cells by multiple signaling pathways. Endocr Relat Cancer. 2010; 17:179-189.

34. Kusakai G, Suzuki A, Ogura T, Miyamoto S, Ochiai A, Kaminishi M, Esumi H. ARK5 expression in colorectal cancer and its implications for tumor progression. Am J Pathol. 2004; 164:987-995.

35. Kubens BS, Zanker KS. Differences in the migration capacity of primary human colon carcinoma cells (SW480) and their lymph node metastatic derivatives (SW620). Cancer Lett. 1998; 131:55-64.

36. Gunasinghe NP, Wells A, Thompson EW, Hugo HJ. Mesenchymal-epithelial transition (MET) as a mechanism for metastatic colonisation in breast cancer. Cancer Metastasis Rev. 2012; 31:469-478. 
37. Chaffer CL, Thompson EW, Williams ED. Mesenchymal to epithelial transition in development and disease. Cells Tissues Organs. 2007; 185:7-19.

38. Hewitt RE, McMarlin A, Kleiner D, Wersto R, Martin P, Tsokos M, Stamp GW, Stetler-Stevenson WG. Validation of a model of colon cancer progression. J Pathol. 2000; 192:446-454.

39. Bu P, Wang L, Chen KY, Rakhilin N, Sun J, Closa A, Tung KL, King S, Kristine Varanko A, Xu Y, Huan Chen J, Zessin AS, Shealy J, et al. miR-1269 promotes metastasis and forms a positive feedback loop with TGF-beta. Nat Commun. 2015; 6:6879.

40. Fabregat I, Malfettone A, Soukupova J. New Insights into the Crossroads between EMT and Stemness in the Context of Cancer. J Clin Med. 2016; 5.

41. Jass JR. Classification of colorectal cancer based on correlation of clinical, morphological and molecular features. Histopathology. 2007; 50:113-130.

42. Horvat M, Stabuc B. Microsatellite instability in colorectal cancer. Radiol Oncol. 2011; 45:75-81.

43. Migliore L, Migheli F, Spisni R, Coppede F. Genetics, cytogenetics, and epigenetics of colorectal cancer. J Biomed Biotechnol. 2011; 2011:792362.
44. Lurje G, Zhang W, Lenz HJ. Molecular prognostic markers in locally advanced colon cancer. Clin Colorectal Cancer. 2007; 6:683-690.

45. Grady WM, Carethers JM. Genomic and epigenetic instability in colorectal cancer pathogenesis. Gastroenterology. 2008; 135:1079-1099.

46. Pires Martins R, Leach RE, Krawetz SA. Whole-body gene expression by data mining. Genomics. 2001; 72:34-42.

47. Barry-Hamilton V, Spangler R, Marshall D, McCauley S, Rodriguez HM, Oyasu M, Mikels A, Vaysberg M, Ghermazien H, Wai C, Garcia CA, Velayo AC, Jorgensen B, et al. Allosteric inhibition of lysyl oxidase-like-2 impedes the development of a pathologic microenvironment. Nat Med. 2010; 16:1009-1017.

48. Musso G, Cassader M, Gambino R. Non-alcoholic steatohepatitis: emerging molecular targets and therapeutic strategies. Nat Rev Drug Discov. 2016; 15:249-274.

49. Tato-Costa J, Casimiro S, Pacheco T, Pires R, Fernandes A, Alho I, Pereira P, Costa P, Castelo HB, Ferreira J, Costa L. Therapy-Induced Cellular Senescence Induces Epithelial-toMesenchymal Transition and Increases Invasiveness in Rectal Cancer. Clin Colorectal Cancer. 2016; 15:170-178 e173. 